\title{
Plasmid-mediated NAD independence in Haemophilus parainfluenzae
}

\author{
Helen M. Windsor, Rosa C. Gromkova* and HendRiK J. KoORNHOF
}

Emergent Pathogen Research Unit of the South African Medical Research Council, School of Pathology of the University of the Witwatersrand and the South African Institute for Medical Research, 7 York Road, Parktown, Johannesburg 2193, South Africa

(Received 25 March 1991; revised 12 June 1991; accepted 20 June 1991)

\begin{abstract}
The location of the genes coding for NAD independence in four unusual clinical isolates of Haemophilus parainfluenzae was determined by transferring these genes to plasmid-free Haemophilus influenzae Rd by transformation and analysing transformants for the presence of plasmids by agarose gel electrophoresis. All NADindependent transformants were found to carry a single plasmid species. The plasmids, originally harboured by the four $H$. parainfluenzae isolates recovered from unrelated sources, were of the same size (5.25 kb). Spontaneous reversion to NAD dependence occurred with a low frequency ( 0.1 to $0.2 \%$ of the progeny of a single clone) in both $H$. parainfluenzae and $H$. influenzae $\mathrm{Rd}$. The revertants had lost this small plasmid. Mitomycin $\mathrm{C}$ exhibited a plasmid 'curing' effect with a frequency of 'curing' of between 1 and $6 \%$ of the surviving clones. It was concluded that the genes conferring NAD independence were located on the small $5.25 \mathrm{~kb}$ plasmid.
\end{abstract}

\section{Introduction}

Most bacteria are able to synthesize NAD (V-factor), which is required for their growth. NAD is a coenzyme necessary for the energy producing processes in both prokaryotic and eukaryotic cells. It also serves as a substrate in some important biological reactions (Foster \& Moat, 1980). Haemophilus parainfluenzae, Haemophilus influenzae and some other Haemophilus species lack the ability to synthesize NAD. These bacteria are able to grow only in media that contain NAD or are supplemented with this growth factor. NAD requirement and requirement for haemin ( $\mathrm{X}$-factor) have been used for many years as a basis for the classification and laboratory identification of Haemophilus species. According to present laboratory practice, isolates belonging to the genus Haemophilus that grow in the absence of NAD cannot be identified as $H$. parainfluenzae or $H$. influenzae.

Recently four naturally occurring NAD-independent $H$. parainfluenzae clinical isolates were identified in our laboratory (Gromkova et al., $1989 a$; Gromkova \& Koornhof, 1990). These strains were recovered from unrelated sources (a genital ulcer, a purulent skin lesion, a sputum specimen and a throat swab). With the exception of their NAD requirement, the strains

\footnotetext{
* Correspondence should be sent to PO Box 1038, Johannesburg 2000 , South Africa.
}

exhibited the biochemical characteristics of $H$. parainfluenzae biotype II. Genetic homology between these unusual strains and a standard strain of $H$. parainfluenzae was confirmed by transformation. The NAD-independent isolates were efficient donors and recipients in transformation of two chromosomal markers, streptomycin resistance and nalidixic acid resistance. It was also found that the marker for NAD independence was transmissible by transformation to typical NAD-requiring $H$. parainfluenzae and $H$. influenzae recipients (Gromkova \& Koornhof, 1990).

In this paper, we provide evidence that the genes conferring NAD independence in $H$. parainfluenzae are located on a small $5.25 \mathrm{~kb}$ plasmid. In addition, we studied some of the properties of this plasmid in two bacterial hosts. Extrachromosomal location of genes involved in NAD biosynthesis has not been reported previously.

\section{Methods}

Bacterial strains and growth. Three strains of NAD-independent $H$. parainfluenzae, designated 9,15 and 19 , were isolated from paediatric patients at Baragwanath Hospital, Johannesburg (Gromkova \& Koornhof, 1990). These were recovered, respectively, from a sputum specimen, a purulent skin lesion and a throat swab. A fourth isolate from a male patient with a genital ulcer who was attending a clinic for sexually transmitted diseases at Carletonville, near Johannesburg, was designated 78 (Gromkova et al., 1989a). H. influenzae Rd and $H$. parainfluenzae 14 (Boss) were provided by G. Leidy, Columbia 
University, New York, USA. Twenty-two $H$. parainfluenzae and five $H$. influenzae strains were isolated from healthy children at a crèche in Johannesburg. Antibiotic-resistant strains, used as donors in transformation, were obtained by selecting spontaneous mutants on antibioticcontaining plates.

Three Escherichia coli strains carrying plasmids pBR322, pUC18 and p7F12 were used as a source of plasmid DNA of known size.

The Haemophilus strains were grown in Brain Heart Infusion (BHI) broth and BHI agar (Becton Dickinson). These media were supplemented with $10 \mu \mathrm{g}$ haemin $\mathrm{ml}^{-1}$ (Sigma) for the $H$. influenzae strains while the NAD-dependent $H$. influenzae and $H$. parainfluenzae strains

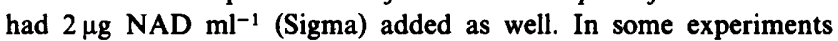
chocolate agar was also used for growth of Haemophilus strains.

DNA preparation. Bacterial DNA was extracted by the method described by Marmur (1961). Plasmid DNA was prepared by the alkaline lysis method described by Sambrook et al. (1989) but with the addition of a phenol/chloroform extraction step. DNA concentration was determined by measuring $A_{260}$.

DNA transformation. Competence was induced by the static aerobic procedure (Gromkova \& Goodgal, 1979; Gromkova et al., 1989 b). For the transformation assay, $0.1 \mathrm{ml}$ purified DNA at a saturating concentration of $1 \mu \mathrm{g} \mathrm{ml}^{-1}$ was added to $0.8 \mathrm{ml}$ BHI broth supplemented with $1 \mathrm{~mm}-\mathrm{MgSO}_{4}$. After the addition of $0.1 \mathrm{ml}$ competent cells, the mixture was incubated for $60 \mathrm{~min}$ at $37^{\circ} \mathrm{C}$ with shaking and then pancreatic DNAase (Sigma) was added to $10 \mu \mathrm{g} \mathrm{ml}^{-1}$. For expression of the genes, the mixture was incubated for $3 \mathrm{~h}$ at $37^{\circ} \mathrm{C}$ and then diluted and plated in triplicate for transformants. Each transformation experiment was repeated three times.

Plasmid characterization. The purified plasmid DNA was separated by agarose gel electrophoresis in $0.8 \%$ agarose using Tris/EDTA/acetate (TEA) buffer in a Bio-Rad Mini-Sub DNA electrophoresis cell. After 90 min at $60 \mathrm{~V}$ the gels were stained with ethidium bromide at a concentration of $0.6 \mu \mathrm{g} \mathrm{ml}^{-1}$. The size of the plasmid conferring NAD independence was determined by comparison with $E$. coli plasmids of known molecular size.

Selection of spontaneous NAD-dependent revertants. A single colony from a BHI agar plate unsupplemented with NAD was subcultured into $10 \mathrm{ml}$ BHI broth in the absence of NAD and was grown overnight by shaking at $37^{\circ} \mathrm{C}$. The culture was diluted and plated on chocolate agar for single colonies. After overnight incubation at $37^{\circ} \mathrm{C}$ about 1000 colonies were examined for NAD requirement by inoculating $1 \mathrm{ml} \mathrm{BHI}$ broth supplemented with NAD and $1 \mathrm{ml} \mathrm{BHI}$ broth unsupplemented with NAD.

Mitomycin C treatment and plasmid 'curing'. A single colony from a BHI agar plate unsupplemented with NAD was subcultured into $10 \mathrm{ml}$ BHI broth in the absence of NAD and grown overnight by shaking at $37^{\circ} \mathrm{C}$. The culture was diluted 1:50 in BHI broth without NAD and grown at $37^{\circ} \mathrm{C}$ with shaking to an $\mathrm{OD}_{650}$ of $0 \cdot 1$. Mitomycin $\mathrm{C}$ (Sigma) was added at different concentrations (see Fig. 4 and Table 4 ) and the culture incubated further. OD readings were recorded at 1 or $2 \mathrm{~h}$ intervals to determine if lysis could be induced.

When plasmid 'curing' was being examined, the culture was grown as described above and after $5 \mathrm{~h}$ an aliquot was diluted, plated onto chocolate agar and incubated at $37^{\circ} \mathrm{C}$. After $24 \mathrm{~h}$ the size and number of colonies were noted and recorded. Approximately 1000 colonies were examined for their NAD requirement as described above.

Plasmid 'curing' with ethidium bromide. A single colony from a BHI agar plate unsupplemented with NAD was inoculated into $10 \mathrm{ml} \mathrm{BHI}$ broth in the absence of NAD and grown overnight by shaking at $37^{\circ} \mathrm{C}$. Tubes with $0.9 \mathrm{ml}$ BHI broth containing ethidium bromide at a final concentration between 0.78 and $100 \mu \mathrm{g} \mathrm{ml}^{-1}$ were inoculated with

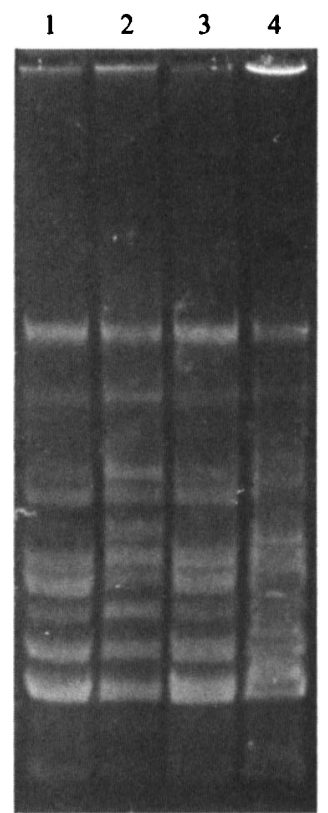

Fig. 1. Agarose gel electrophoresis of plasmid DNA preparations from NAD-independent $H$. parainfluenzae strains. Lanes: 1 , isolate $9 ; 2$, isolate $15 ; 3$, isolate $19 ; 4$, isolate 78 .

$0.1 \mathrm{ml}$ of the culture and incubated overnight at $37^{\circ} \mathrm{C}$. Cultures that showed minimal inhibition of growth were diluted and plated onto chocolate agar for single colonies. About 1000 colonies were tested for NAD requirement.

\section{Results}

\section{Presence of plasmids in NAD-independent $H$. parainfluenzae}

In order to study whether or not the genes conferring NAD independence were located extrachromosomally, DNA preparations from the four naturally occurring NAD-independent $H$. parainfluenzae isolates were examined for the presence of plasmids by agarose gel electrophoresis (Fig. 1). Numerous extrachromosomal bands were present in these preparations, suggesting that each $H$. parainfluenzae strain carried several plasmids. To see whether the genes coding for NAD independence resided on one of these plasmids, we compared the transforming activity of the plasmid DNA preparation with total bacterial DNA using NAD independence as a putative plasmid marker and streptomycin resistance $\left(\mathrm{Str}^{\mathrm{r}}\right)$ as a known chromosomal marker. The results presented in Table 1 show that plasmid DNA was more effective than bacterial DNA in transforming $H$. parainfluenzae 14 competent cells to NAD independence whilst bacterial DNA was about 1000 -fold more effective than plasmid DNA in transforming the same competent cells to $\mathrm{Str}^{\mathrm{r}}$. Transformation experiments 
Table 1. Transformation of $H$. parainfluenzae 14 by bacterial and plasmid DNA from NAD-independent $H$. parainfluenzae

The transformation procedure is described in Methods. The concentration of streptomycin used for selection of transformants was $100 \mu \mathrm{g} \mathrm{ml} \mathrm{m}^{-1}$.

\begin{tabular}{|c|c|c|c|c|}
\hline \multirow[b]{3}{*}{ DNA donor } & \multicolumn{4}{|c|}{ Number of transformants per $5 \times 10^{8}$ c.f.u. $\mathrm{ml}^{-1}$} \\
\hline & \multicolumn{2}{|c|}{ Total bacterial DNA } & \multicolumn{2}{|c|}{ Plasmid DNA } \\
\hline & $\begin{array}{c}\text { NAD } \\
\text { independence }\end{array}$ & Str ${ }^{r}$ & $\begin{array}{c}\text { NAD } \\
\text { independence }\end{array}$ & Str ${ }^{r}$ \\
\hline $\begin{array}{l}\text { H. parainfluenzae } 9 \mathrm{Str}^{r} \\
\text { H. parainfluenzae } 15 \mathrm{Str}^{r} \\
\text { H. parainfluenzae } 19 \mathrm{Str}^{r} \\
\text { H. parainfluenzae } 78 \mathrm{Str}^{r}\end{array}$ & $\begin{array}{l}8.1 \times 10^{4} \\
6.2 \times 10^{4} \\
8.9 \times 10^{4} \\
9.1 \times 10^{4}\end{array}$ & $\begin{array}{l}1.8 \times 10^{6} \\
1.4 \times 10^{6} \\
2.1 \times 10^{6} \\
1.9 \times 10^{6}\end{array}$ & $\begin{array}{l}5.5 \times 10^{5} \\
3.3 \times 10^{5} \\
4.8 \times 10^{5} \\
5.2 \times 10^{5}\end{array}$ & $\begin{array}{l}4.4 \times 10^{2} \\
3.2 \times 10^{2} \\
3.9 \times 10^{2} \\
4.8 \times 10^{2}\end{array}$ \\
\hline
\end{tabular}

Table 2. Transformation of $H$. influenzae $R d$ by plasmid DNA from $N A D$-independent $H$. parainfluenzae

\begin{tabular}{lcr}
\hline \hline & \multicolumn{2}{c}{$\begin{array}{c}\text { No. of transformants per } \\
5 \times 10^{8} \text { c.f.u. ml }\end{array}$} \\
\cline { 2 - 3 } \multicolumn{1}{c}{ DNA donor } & NAD independence & Str $^{-1}$ \\
\hline H. parainfluenzae 9 $\mathrm{Str}^{\mathrm{r}}$ & $1.8 \times 10^{4}$ & 8 \\
H. parainfluenzae 15 $\mathrm{Str}^{\mathrm{r}}$ & $2.1 \times 10^{4}$ & 9 \\
H. parainfluenzae 19 $\mathrm{Str}^{\mathrm{r}}$ & $9.8 \times 10^{3}$ & 7 \\
H. parainfluenzae 78 $\mathrm{Str}^{\mathrm{r}}$ & $1.9 \times 10^{4}$ & 9 \\
\hline \hline
\end{tabular}

were repeated using three different batches of competent cells and these yielded similar results, supporting the conclusion that the genes coding for NAD independence were probably plasmid-linked.

\section{Presence of plasmids in NAD-independent transformants of $H$. influenzae $R d$}

To provide direct evidence that NAD independence was controlled by genes located on a plasmid, we attempted to transfer these genes to a plasmid-free recipient. We were, however, not able to find a plasmid-free $H$. parainfluenzae strain. The standard, highly transformable $H$. parainfluenzae 14 is known to carry several plasmids (Mann \& Rao, 1979) and was therefore not a suitable recipient, nor were 22 local strains of $H$. parainfluenzae recovered from healthy children (data not presented). Previous studies have shown that NAD independence can be transmitted by transformation to the standard, highly transformable $H$. influenzae $\mathrm{Rd}$ strain (Gromkova \& Koornhof, 1990), which has been shown to be plasmid-free. Results from transformation experiments using, as donor DNA, plasmid DNA isolated from the NAD-independent $H$. parainfluenzae isolates to $H$. influenzae $\mathrm{Rd}$ competent cells, are given in Table 2. Five NAD-independent transformants from

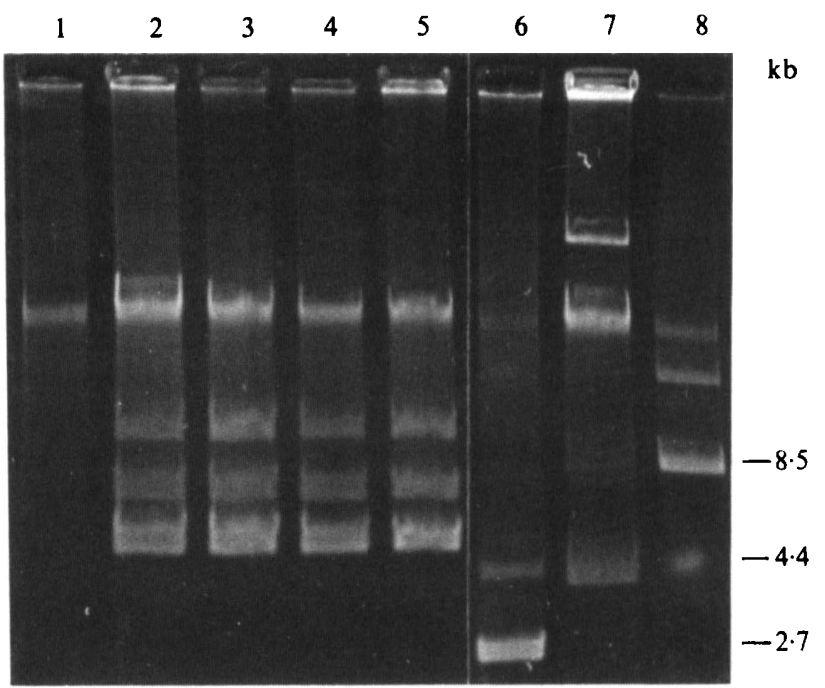

Fig. 2. Agarose gel electrophoresis of plasmid DNA preparations from NAD-independent $\boldsymbol{H}$. influenzae Rd transformants. Lanes: 1, plasmidfree $H$. influenzae Rd strain used as transformation recipient; 2 to 5 , transformants of $\boldsymbol{H}$. influenzae Rd using $H$. parainfluenzae isolates 9,15 , 19 and 78, respectively, as DNA donors; 6 to 8 plasmid markers pUC18, pBR322 and p7F12, respectively.

each donor DNA were examined for the presence of plasmids and all transformants were found to carry a single plasmid. Fig. 2 shows agarose gel electrophoresis of one representative NAD-indępendent transformant from each group (lanes 2 to 5 ). The electrophoretic mobility of DNA preparations from the plasmid-free $H$. influenzae $\mathrm{Rd}$ strain that was used as a recipient in transformation is shown in lane 1 . The single band present in this preparation corresponds to chromosomal DNA. Three extrachromosomal bands were present in each of the NAD-independent $H$. influenzae Rd transformants (lanes 2 to 5) corresponding to the three molecular forms of plasmid DNA : covalently closed circular, open circular and linear. The plasmids originally harboured by 
Table 3. Spontaneous loss of NAD independence in $H$. parainfluenzae and in $H$. influenzae $R d$

Approximately 1000 clones from each strain were tested for their NAD requirement.

\begin{tabular}{lc}
\hline \hline \multicolumn{1}{c}{ Strain } & $\begin{array}{c}\text { Percentage revertants } \\
\text { to NAD dependence }\end{array}$ \\
\hline H. parainfluenzae 9 & $0 \cdot 12$ \\
H. parainfluenzae 15 & $0 \cdot 14$ \\
H. parainfluenzae 19 & $0 \cdot 16$ \\
H. parainfluenzae 78 & $0 \cdot 19$ \\
H. influenzae Rd 9 & $0 \cdot 15$ \\
H. influenzae Rd 15 & $0 \cdot 21$ \\
H. influenzae Rd 19 & $0 \cdot 13$ \\
H. influenzae Rd 78 & $0 \cdot 22$ \\
\hline \hline
\end{tabular}

the four NAD-independent $H$. parainfluenzae isolates, which were recovered from unrelated sources, appear to be of identical size. By comparing the electrophoretic mobility of three standard plasmids of known size (Fig. 2, lanes 6 to 8 ) and the mobility of the plamids from the NAD-independent $H$. influenzae $\mathrm{Rd}$ transformants, these latter plasmids were estimated to be $5 \cdot 25 \mathrm{~kb}$.

To exclude the possibility of accidental co-transfer of a small highly transformable plasmid with the genes coding for NAD independence, 10 transformants selected for a chromosomal genetic marker $\left(\mathrm{Str}^{\mathrm{r}}\right)$ were tested for the presence of plasmids. None of them was found to carry a small plasmid. These results show that only transformants selected for NAD independence acquired the plasmid and indicate that the genetic element coding for NAD independence is associated with the plasmid.

\section{Stability of NAD independence in $H$. parainfluenzae and $H$. influenzae}

Since plasmid genes are known to be less stable than chromosomal genes, we examined the four naturally occurring NAD-independent $\boldsymbol{H}$. parainfluenzae isolates and the NAD-independent transformants of $H$. influen$z a e \mathrm{Rd}$ for their ability to revert spontaneously to NAD dependence. The results presented in Table 3 show that spontaneous loss of NAD independence occurred with a low frequency in both $H$. parainfluenzae and $H$. influenzae hosts. The colony size of the NAD-dependent revertants was smaller than that of the NAD-independent clones when plated on chocolate agar. Four NAD-requiring revertants obtained from NAD-independent transformants of $H$. influenzae $\mathrm{Rd}$ were examined for the presence of plasmids by gel electrophoresis (Fig. 3, lanes 1 to 4). The results show that the reversion to NAD dependence corresponded with the loss of the small $5.25 \mathrm{~kb}$ plasmid coding for NAD independence (lane 5).

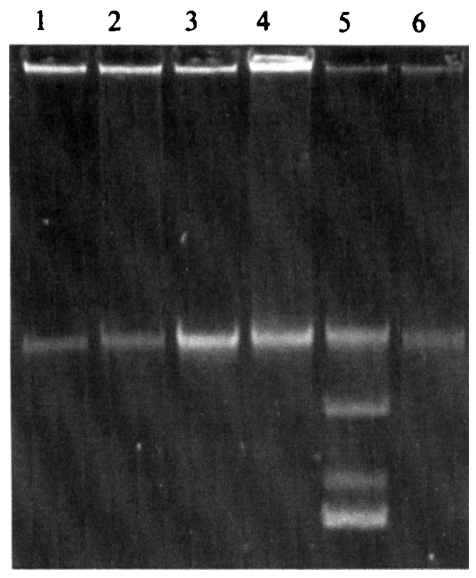

Fig. 3. Agarose gel electrophoresis of plasmid DNA preparations from NAD-dependent spontaneous revertants of NAD-independent $\boldsymbol{H}$. influenzae $\mathrm{Rd}$ transformants. Lanes: 1 to 4, spontaneous revertants of . $H$. influenzae $\mathrm{Rd}$ transformants by $H$. parainfluenzae DNA from isolates 9, 15, 19 and 78, respectively; 5, NAD-independent $H$. influenzae $\mathrm{Rd}$ transformant ( $H$. parainfluenzae 9 as DNA donor); 6 , plasmid-free $H$. influenzae $\mathrm{Rd}$.

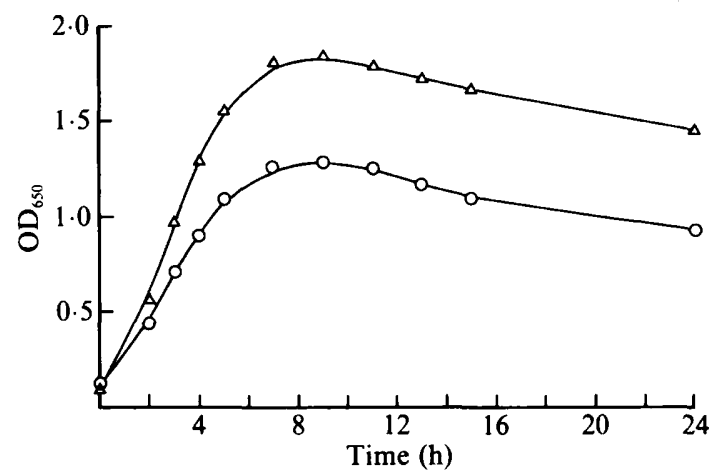

Fig. 4. Lytic curves of NAD-independent $H$. parainfluenzae 9 in BHI broth in the presence and absence of mitomycin C. $\triangle$, No additives; $\mathrm{O}$, addition of $0.2 \mu \mathrm{g}$ mitomycin $\mathrm{C} \mathrm{ml}^{-1}$.

\section{Treatment of NAD-independent $H$. parainfluenzae and} $H$. influenzae with mitomycin $C$

Each of the four naturally occurring NAD-independent $H$. parainfluenzae isolates exhibited spontaneous lysis after overnight culture in BHI broth and their NADdependent spontaneous revertants did not show loss of this lytic activity. Assuming that the presence of a prophage may be responsible for this, we attempted to induce lysis by treatment with mitomycin $\mathrm{C}$. The lytic curves of the NAD-independent $H$. parainfluenzae isolates follow the profiles of those of $H$. parainfluenzae 9 presented in Fig. 4. The inability of mitomycin $\mathrm{C}$ to stimulate lysis suggested that these strains may carry non-inducible prophages. This prompted us to search for a phage-sensitive indicator strain but all $22 \mathrm{H}$. parain- 
Table 4. 'Curing' of NAD independence in $H$. parainfluenzae and in $H$. influenzae $R d$ by mitomycin $C$

Mitomycin C concentration was $0.2 \mu \mathrm{g} \mathrm{ml}^{-1}$ for $H$. parainfluenzae and $0.1 \mu \mathrm{g} \mathrm{ml}^{-1}$ for $H$. influenzae $\mathrm{Rd}$.

\begin{tabular}{lc}
\hline \multicolumn{1}{c}{ Strain } & $\begin{array}{c}\text { Percentage } \\
\text { NAD-dependent clones* }\end{array}$ \\
\hline H. parainfluenzae 9 & $3 \cdot 17$ \\
H. parainfluenzae 15 & $6 \cdot 05$ \\
H. parainfluenzae 19 & 4.42 \\
H. parainfluenzae 78 & $4 \cdot 13$ \\
H. influenzae $\mathrm{Rd} 9$ & 1.30 \\
H. influenzae Rd 15 & 1.00 \\
H. influenzae Rd 19 & 0.59 \\
H. influenzae Rd 78 & 0.82 \\
\hline \hline
\end{tabular}

* The survival rate of treated $H$. parainfluenzae and $H$. influenzae $R d$ strains was 0.1 to $0.5 \%$. About 1000 surviving clones were tested for their NAD requirement.

fluenzae and $5 \mathrm{H}$. influenzae strains tested failed to produce phage plaques.

Although lysis was not stimulated by mitomycin $\mathrm{C}$ we noticed an increase in the number of small-size colonies when determining the survival rate of the treated cultures. About 0.1 to $0.5 \%$ of the bacteria survived using the mitomycin $\mathrm{C}$ concentrations shown in Table 4. Reversion to NAD dependence in both $H$. parainfluenzae and $H$. influenzae Rd was enhanced 4- to 40 -fold after treatment with mitomycin $C$ (Table 4) as compared to the spontaneous rate (Table 3). The results in Table 4 show that the 'curing' effect of mitomycin $\mathrm{C}$ was stronger in $H$. parainfluenzae than in $H$. influenzae. Similar results were obtained when higher $\left(0.2 \mu \mathrm{g} \mathrm{ml}^{-1}\right)$ and lower $\left(0.05 \mu \mathrm{g} \mathrm{ml}^{-1}\right)$ concentrations of mitomycin $C$ were used. Four NAD-requiring revertants obtained after mitomycin $\mathrm{C}$ treatment were examined for the presence of plasmids and were found to be plasmid-free (data not presented).

We were not able to enhance the frequency of reversion to NAD dependence of either $H$. parainfluen$z a e$ or $H$. influenzae $\mathrm{Rd}$ by treatment with ethidium bromide, which has been found to be an effective plasmid 'curing' agent in many bacterial systems (Bouanchaud et al., 1969).

\section{Discussion}

The isolation from natural sources of four unusual $H$. parainfluenzae strains that were able to grow in the absence of NAD raised the question of the genetic basis of this property. In order to address this question, we attempted to determine whether the genes conferring NAD independence were located on the chromosome or on an extrachromosomal genetic element. The inability to induce NAD-independent mutants of $H$. parainfluen$z a e$ by treatment with nitrosoguanidine (R. Gromkova, unpublished data) and the significant difference in the efficiencies of transformation of the marker for NAD independence and those of two chromosomal genetic markers, $\operatorname{Str}^{\mathrm{r}}$ and nalidixic acid resistance (Gromkova \& Koornhof, 1990), suggested that the genes for NAD independence may reside extrachromosomally. Marker efficiencies of chromosomal markers in $H$. parainfluen$z a e$ and $H$. influenzae do not vary by more than a factor of two in intraspecific transformation (Goodgal \& Gromkova, 1973). Previous studies have demonstrated that a plasmid conferring ampicillin resistance in $H$. parainfluenzae was about 100-fold less efficient in transformation than the chromosomal $\mathrm{Str}^{\mathrm{r}}$ marker when $\mathrm{CsCl}$ gradient-purified plasmid RSF0885 DNA was used (Gromkova \& Goodgal, 1979).

In the present study, transformation to NAD independence of $H$. parainfluenzae 14 competent cells by total bacterial DNA was about 20 -fold less efficient than transformation by the chromosomal Str ${ }^{\mathrm{r}}$ marker (Table 1). Plasmid DNA preparations from the four naturally occurring $H$. parainfluenzae strains were more efficient than total bacterial DNA in transforming $H$. parainfluenzae 14 to NAD independence, while the plasmid DNA preparations transformed the chromosomal Str ${ }^{r}$ marker far less efficiently than bacterial DNA (Table 1). These findings provided further evidence in support of the plasmid hypothesis. The residual transforming activity to $\operatorname{Str}^{\mathrm{r}}$ in the plasmid preparations was probably due to incomplete removal of chromosomal DNA. One should bear in mind that the $H$. parainfluenzae plasmid preparations used in the transformation experiment each contained several plasmids (Fig. 1). If purified plasmid DNA containing only one plasmid species were used, one would expect higher transforming efficiency to NAD independence because of the lack of competition from other DNA molecules for DNA-binding sites.

Direct evidence that the genes conferring NAD independence were plasmid-linked was provided by the finding that after transforming the plasmid-free $H$. influenzae Rd to NAD independence, all the transformants acquired a small $5.25 \mathrm{~kb}$ plasmid (Fig. 2). Although the size of this plasmid was similar to that of plasmid RSF0885 conferring ampicillin resistance (de Graaff et al., 1976), the plasmid coding for NAD independence transformed cells with a higher frequency than the former plasmid (Gromkova \& Goodgal, 1979). This was probably due to the more efficient expression of the marker for NAD independence than that of the $\beta$ lactamase marker. Because of its high transformability and the presence of a biological marker that can be expressed and selected easily, the plasmid conferring 
NAD independence has the potential to become a good cloning vector in Haemophilus.

The similarity in size of the plasmids conferring NAD independence, despite the fact that they have originated from four $H$. parainfluenzae strains isolated from unrelated sources, suggested that they may have a common origin. It is possible that the plasmids have been acquired by the four strains independently by genetic exchange with an organism able to synthesize NAD. However, since the NAD-independent isolates of $H$. parainfluenzae were all recovered in or near Johannesburg the clonal distribution of a single unusual NADindependent $H$. parainfluenzae strain cannot be excluded. In this regard it is interesting that the NAD-independent $H$. parainfluenzae isolates all belong to the same biotype (Gromkova \& Koornhof, 1990) and that each strain carries several plasmids with similar electrophoretic mobility (Fig. 1). In addition, the four strains were able to spontaneously undergo partial lysis (Fig. 4). These strains may have a survival advantage as a result of their ability to synthesize NAD.

The relative instability of NAD independence in both $H$. parainfluenzae and $H$. influenzae, which is due to the loss of the $5.25 \mathrm{~kb}$ plasmid (Fig. 3), is characteristic of plasmid DNA.

There have been no previous reports on the extrachromosomal location of genes involved in NAD biosynthesis. The only genes that have so far been found to be located on plasmids in Haemophilus species are those conferring drug resistance. In most bacterial systems, genes coding for metabolic functions that are essential for the survival of the cells do not reside on plasmids but on the chromosome. However, this does not apply to $H$. parainfluenzae, as wild-type $H$. parainfluenzae strains are usually not able to synthesize NAD. It appears unlikely that the genes located on the plasmid code for de novo synthesis of NAD because of the small size of this plasmid; for example, in E. coli the de novo synthesis of NAD involves at least six steps (Foster \& Moat, 1980). Probably, the biosynthetic pathway of NAD in these $H$. parainfluenzae strains involves the use of a pyridine intermediate similar to that in other NAD-independent Haemophilus species (Kasarov \& Moat, 1973; Cynamon et al., 1988; Niven \& Lévesque, 1988).

An interesting observation was that mitomycin $\mathrm{C}$ can be used as a plasmid 'curing' agent in both $H$. parainfluenzae and $H$. influenzae. Mitomycin $\mathrm{C}$ is an antibiotic that has bactericidal and mutagenic effects on bacteria (Iijima \& Hagiwara, 1960) due to structural changes in DNA (Waring, 1968). It has the ability to block selectively the synthesis of host DNA but to permit viral DNA synthesis. Furthermore, mitomycin $C$ has been used extensively as an effective prophage inducer (Otsuji et al., 1959) and it is also able to induce the production of some colicins (Hardy \& Meynell, 1972). The demonstration that NAD-dependent revertants of $H$. influenzae Rd transformants obtained after treatment with mitomycin $\mathrm{C}$ failed to show the presence of plasmids indicates that the loss of NAD independence was not due to a mutagenic effect of mitomycin $C$ but to its 'curing' effect. The latter effect was probably due to selective inhibition of plasmid DNA without inactivation of the host DNA. In $H$. influenzae the plasmid 'curing' by mitomycin $\mathrm{C}$ appears to be less efficient than in $H$. parainfluenzae (Table 4). This was probably due to the stronger bactericidal effect of mitomycin $\mathrm{C}$ on $H$. influenzae. There are no previous reports on the use of mitomycin $\mathrm{C}$ as a plasmid 'curing' agent in Haemophilus but mitomycin $\mathrm{C}$ has been widely used in Pseudomonas species 'curing' catabolic plasmids (Chakrabarty, 1972; Williams \& Worsey, 1976).

There is no indication that the spontaneous lysis of the NAD-independent $H$. parainfluenzae was associated with the presence of a prophage. It was also evident that the genes coding for the lytic activity were not linked to the plasmid conferring NAD independence because, after spontaneous loss of the plasmid, the NADdependent revertants were still able to undergo spontaneous lysis.

\section{References}

Bouanchaud, D. H., Scavizzi, M. R. \& Chabbert, Y. A. (1969). Elimination by ethidium bromide of antibiotic resistance in enterobacteria and staphylococci. Journal of General Microbiology 54, 417-425.

Chakrabarty, A. M. (1972). Genetic basis of the biodegradation of salicylate in Pseudomonas. Journal of Bacteriology 112, 815-823.

Cynamon, M. H., Sorg, T. B. \& Patapow, A. (1988). Utilization and metabolism of NAD by Haemophilus parainfluenzae. Journal of General Microbiology 134, 2789-2799.

Foster, J. W. \& MOAT, A. G. (1980). Nicotinamide adenine dinucleotide biosynthesis and pyridine nucleotide cycle metabolism in microbial systems. Microbiological Reviews 44, 83-105.

GoOdGal, S. H. \& Gromkova, R. (1973). The biological specificity of Haemophilus endodeoxyribonucleases which attack heterologous DNA. In Bacterial Transformation, pp. 743-758. Edited by L. Archer. London \& New York: Academic Press.

De GraAfF, J., Elwell, L. P. \& FAlKow, S. (1976). Molecular nature of two beta-lactamase-specifying plasmids isolated from Haemophilus influenzae type b. Journal of Bacteriology 126, 439-446.

Gromkova, R. \& Goodgal, S. H. (1979). Transformation by plasmid and chromosomal DNA in Haemophilus parainfluenzae. Biochemical and Biophysical Research Communications 88, 1428-1434.

Gromkova, R. \& KOORNHOF, H. J. (1990). Naturally occurring NADindependent Haemophilus parainfluenzae. Journal of General Microbiology 136, 1031-1035.

Gromkova, R., DANGOR, Y. \& MILLER, S. D. (1989a). V-factor (NAD) independent Haemophilus parainfluenzae recovered from a human genital ulcer. European Journal of Clinical Microbiology and Infectious Diseases 8, 569.

Gromkova, R., Rowji, P. B. \& KoORnhof, H. J. (1989b). Induction of competence in nonencapsulated and encapsulated strains of Haemophilus influenzae. Current Microbiology 19, 241-245. 
Hardy, K. G. \& MeYnell, G. G. (1972). Colicin factors and mitomycin-C. Journal of General Microbiology 73, 547-549.

IIIMA, T. \& HaGiWARA, A. (1960). Mutagenic action of mitomycin C on Escherichia coli. Nature, London 185, 395-396.

KASAROV, L. B. \& MOAT, A. G. (1973). Biosynthesis of NAD in Haemophilus haemoglobinophilus. Biochimica et Biophysica Acta 320, 372-378.

MANN, M. B. \& RAO, R. N. (1979). Characterization of plasmids from Haemophilus parainfluenzae and Haemophilus haemolyticus. Plasmid 2, 503-506.

MARMUR, J. (1961). A procedure for the isolation of deoxyribonucleic acid from microorganisms. Journal of Molecular Biology 3, 208-218.

Niven, D. F. \& Lévesque, M. (1988). V-factor-dependent growth of Actinobacillus pleuropneumoniae biotype 2 (Bertschinger 2008/76). International Journal of Systematic Bacteriology 38, 319-320.
OTsuj, N., Sekiguchi, M., InIMA, T. \& TEKaGI, Y. (1959). Induction of phage formation in the lysogenic Escherichia coli $\mathrm{K} 12$ by mitomycin C. Nature, London 184, 1079-1080.

Sambrook, J., Fritsch, E. F. \& Manitis, T. (1989). Molecular Cloning: a Laboratory Manual, 2nd edn. Cold Spring Harbor, NY: Cold Spring Harbor Laboratory.

W ARING, M. J. (1968). Drugs which affect the structure and function of DNA. Nature, London 219, 1320-1325.

Williams, P. A. \& Worsey, M. J. (1976). Ubiquity of plasmids in coding for toluene and xylene metabolism in soil bacteria: evidence for the existence of new TOL plasmids. Journal of Bacteriology 125, 818-828. 